\title{
Axillary lymph node dissection for patients with invasive breast cancer at Charlotte Maxeke and Chris Hani Baragwanath Academic Hospitals
}

\author{
C Groenewald, ${ }^{1}$ H Cubasch, ${ }^{1}$ A Mannell, ${ }^{2}$ O Ayeni, ${ }^{3}$ S Nietz ${ }^{4}$ \\ ${ }^{1}$ Department of Surgery, University of the Witwatersrand, Chris Hani Baragwanath Academic Hospital, South Africa \\ ${ }^{2}$ Department of Surgery, University of the Witwatersrand, South Africa \\ ${ }^{3}$ Research Unit, Faculty of Health Sciences, University of the Witwatersrand, South Africa \\ ${ }^{4}$ Department of Surgery, University of the Witwatersrand, Charlotte Maxeke Johannesburg Academic Hospital, South Africa
}

Corresponding author: Dr Carolette Groenewald (pennycaro@gmail.com)

\begin{abstract}
Background: The extent of axillary surgery correlates with its morbidity and sentinel lymph node biopsy (SLNB) has become the standard of care in clinically node-negative (cN0) patients. This study aims to evaluate the application of SLNB and axillary lymph node dissection (ALND) and the associated risk factors for node-negative ALND in our units.

Methods: We included female patients with primary breast cancer who underwent axillary surgery in the breast units at Charlotte Maxeke Johannesburg Academic Hospital and Chris Hani Baragwanath Academic Hospital from March 2013 to March 2015. Univariate and multivariable logistic regression models were used to determine factors associated with pathological node-negative (pN0) ALND.

Results: 505 patients were included and 344 patients were staged clinically node-positive (68.1\%), 161 (31.9\%) were assessed as clinically node-negative and deemed eligible for SLNB. Sensitivity of clinical nodal staging was $85.9 \%$ with a positive predictive value of $76.5 \%$. The majority of patients $(313,61.9 \%)$ underwent primary surgery while $192(38.1 \%)$ underwent surgery after NACT. We performed 118 SLNBs and 387 ALNDs of which 97 were pathologically node-negative. Risk was not increased after NACT (OR 1.06, $\mathrm{p}=0.790)$. We identified a significant risk in patients with triple-negative and HER-2 enriched subtypes compared to hormone receptor-positive patients (OR 3.05, 95\% CI: 1.6-5.7, p = 0.001 and OR 2.25, 95\% CI: $1.1-4.8, \mathrm{p}=0.035)$.

Conclusions: The prevalence of pN0 ALND was 25.06\%. In our cohort a significantly higher risk was found in hormone receptor-negative tumours.

Preoperative nodal assessment needs to be optimised and include pathological confirmation. SLNB needs to be extended to patients after NACT despite resource-constraints.

Keywords: axillary lymph node dissection, invasive breast cancer, Charlotte Maxeke Johannesburg Academic Hospital, Chris Hani Baragwanath Academic Hospital
\end{abstract}

S Afr J Surg 2019;57(4) http://dx.doi.org/10.17159/2078-5151/2019/v57n4a3007

\begin{tabular}{|c|c|c|c|}
\hline \multicolumn{4}{|c|}{ Nomenclature } \\
\hline CHBAH & I - Chris Hani Baragwanath Academic Hospital & PR & - Progesterone receptor \\
\hline $\mathrm{cN}+$ & - Clinically node-positive & HER-2 & - Human epidermal growth factor receptor 2 \\
\hline FNA & - Fine needle aspiration & pN0 & - Pathological node-negative \\
\hline ALND & - Axillary lymph node dissection & $\mathrm{pN}+$ & - Pathological node-positive \\
\hline SLNB & - Sentinel lymph node biopsy & cN0 & - Clinically node-negative \\
\hline NACT & - Neoadjuvant chemotherapy & PPV & - Positive predictive value \\
\hline CMJAH & - Charlotte Maxeke Johannesburg Academic Hospital & NPV & - Negative predictive value \\
\hline ER & - Estrogen receptor & OR & - Odds ratio \\
\hline
\end{tabular}




\section{Introduction}

The axillary lymph node status forms part of tumour staging and is an important prognostic factor for patients with invasive breast cancer. ${ }^{1,2}$ Methods to stage the axilla include physical examination, dedicated imaging and histopathological confirmation. Clinical evaluation of the axilla by palpation alone is relatively inaccurate and has a reported sensitivity of $35 \% .^{3}$ Axillary lymph nodes may appear clinically and radiologically enlarged due to causes other than breast cancer. HIV infection is commonly associated with benign axillary lymphadenopathy and recent data from Chris Hani Baragwanath Academic Hospital (CHBAH) showed that $19.7 \%$ of patients with breast cancer tested positive for HIV. ${ }^{4}$ The term "clinically node-positive" has been redefined over the past years and in current practice it usually entails preoperative confirmation by cytology or core biopsy. In a meta-analysis the median sensitivity of ultrasound was found to be $61.4 \%$ and the specificity $82 \%$, these values increased further to $79.4 \%$ and $100 \%$ with the addition of ultrasoundguided node biopsy. ${ }^{5}$ In patients who appear clinically node-positive $(\mathrm{cN}+)$ due to palpable and/or sonographically suspicious lymph nodes, fine needle aspiration (FNA) or core needle biopsy of a suspicious lymph node can confirm nodal involvement and identify patients who may proceed directly to axillary lymph node dissection (ALND) rather than sentinel lymph node biopsy (SLNB). In cases where the FNA is negative the patient will undergo SLNB. ${ }^{6}$ Unfortunately, we could not implement this approach in our institutions to date due to resource constraints and patients who have palpable lymphadenopathy or sonographically suspicious nodes proceed directly to ALND.

ALND carries morbidity and lymphoedema of the arm is the most frequent long-term complication. $^{7}$ Since it is directly related to the extent of surgery, SLNB has become the standard of care in patients who have no nodal involvement. ALND has no oncological benefit in node-negative patients and is thus difficult to justify. ${ }^{8}$ An aspect that has been recently evolving is the use of SLNB following neoadjuvant chemotherapy (NACT). The SLNB method has been adopted as an axilla-conserving option in the NCCN guidelines for patients who were initially $\mathrm{cN}+$, but who are down-staged following NACT to clinically node-negative (cN0) disease. ${ }^{9}$ This was not adopted in our practice at the time of this study.

In view of the general trend towards axilla-preservation, we audited the use of SLNB and ALND in two breast units and evaluate risk factors for a negative dissection. There have been no previous studies from South Africa to date.

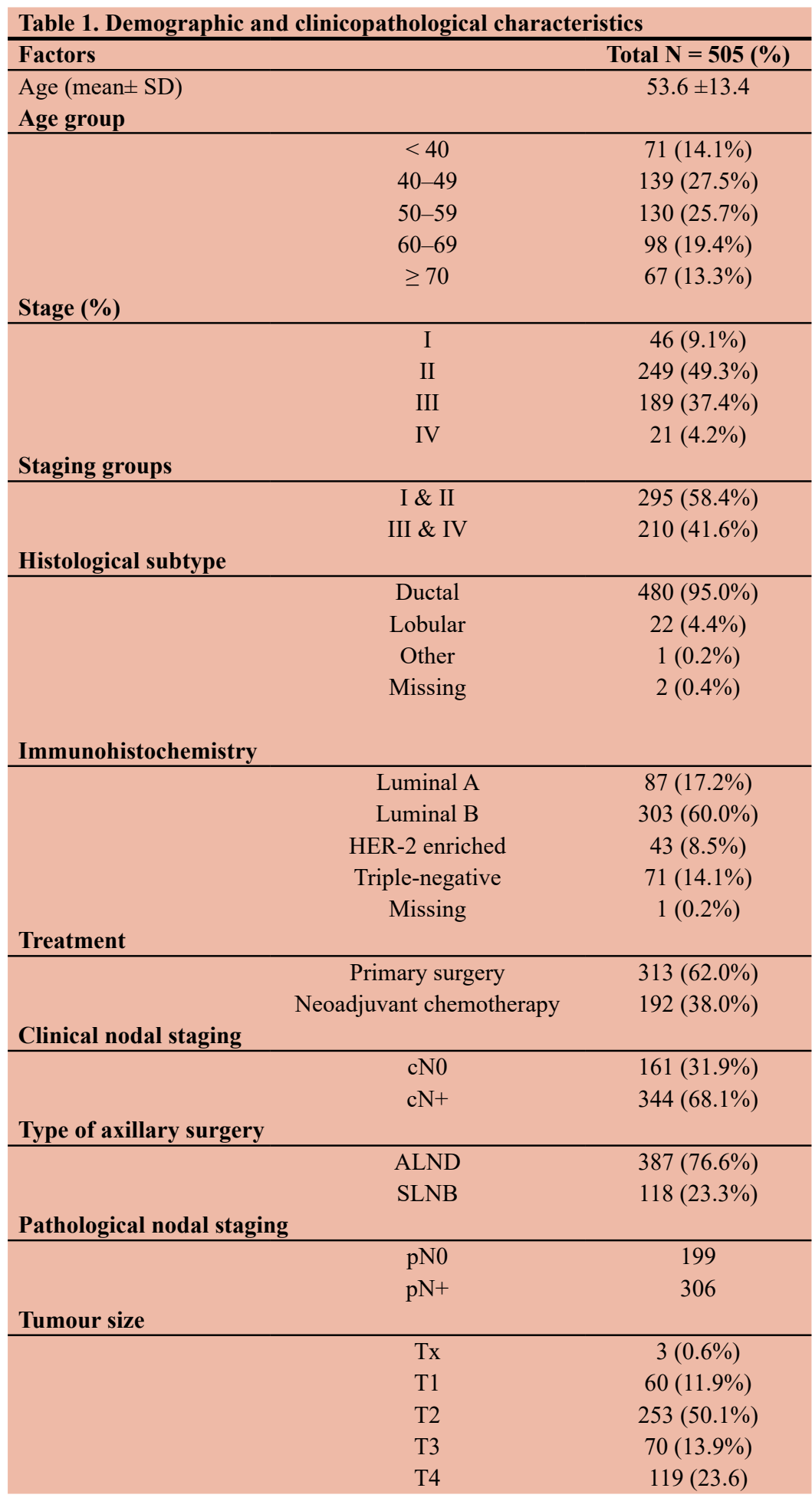

\section{Methods}

We performed a retrospective review at Charlotte Maxeke Johannesburg Academic Hospital (CMJAH) and Chris Hani Baragwanath Academic Hospital (CHBAH) breast units. Patients who presented with primary invasive breast cancer and who underwent axillary surgery from March 2013 to March 2015 were included. Patients were recruited via theatre and admission records at $\mathrm{CMJAH}$ and via the electronic database at CHBAH. 
Data were extracted from histology records, patient records via the breast files at CMJAH and the database at CHBAH. Variables extracted included: site, age, TNM staging at presentation, preoperative histological type of tumour, immunohistochemistry, preoperative histological confirmation of axillary metastasis where available, type of axillary surgery performed, timing of surgery and the final postoperative histopathology report.

Males, incomplete records, patients with recurrent breast cancer and non-invasive breast cancer as well as cases where invasive breast cancer was not primary presentation were excluded.

Intrinsic subtypes were determined by immunohistochemistry according to the following criteria: Luminal A: Estrogen receptor (ER) and/or Progesterone receptor (PR) positive, Human epidermal growth factor 2 (HER-2) negative and Ki-67 < 15; Luminal B: ER and/or PR positive, Ki-67 $\geq 15$ or ER and/or PR positive with HER-2 positive; HER2 enriched: HER-2 positive alone (ER and PR negative); Triple-negative: ER negative, PR negative, HER-2 negative. ${ }^{10}$ Patients were grouped according to preoperative and postoperative node status.

Nodal status was determined by clinical examination as well as radiological evaluation of axillary lymph nodes by ultrasound. Features such as loss of reniform shape of the node, absence of the fatty hilum and increased cortical thickening more than $3 \mathrm{~mm}$ suggest lymph node metastasis. ${ }^{11}$ Descriptive statistics were performed reporting percentages for categorical variables, means and medians for continuous variables. To determine factors associated with pathological node-negative (pN0) ALND, we implemented univariate and multivariate logistic regression models. All variables that were significant at $\mathrm{p}<0.1$ in univariate analysis were evaluated in the multivariate analysis and non-significant factors were dropped with stepwise backward regression. A two-sided p-value of $<0.05$ was considered significant throughout. A goodness of fit test was carried out on the final model to determine how well the model fits into the set of variables. All available data were used for each univariate analysis. In the multivariable analysis, patients with missing data for included variables were dropped from the model. Analysis was carried out using Stata version 14 (StataCorp Limited, Texas, United States of America).

Ethics approval was obtained from the Human Research Ethics Committee (Medical) of the University of the Witwatersrand prior to the commencement of this study (MP150549).

\section{Results}

A total number of 624 patients underwent axillary surgery at CMJAH and CHBAH breast units from March 2013 to March 2015. We excluded 119 patients according to our predefined criteria and identified 505 eligible patients for our study (Figure 1). The main reasons for exclusion were recurrent disease and absence of invasive cancer.

The mean age was 53.6 (SD 13.4) years. 46 patients presented with clinical stage 1 disease (9.1\%), 249 (49.31\%) presented as stage 2, $189(37.43 \%)$ were stage 3 and 21 $(4.16 \%)$ stage 4 . Most of the patients $(480,95.1 \%)$ were diagnosed ductal carcinoma, $22(4.4 \%)$ lobular carcinoma, one $(0.2 \%)$ had a mixed type and two $(0.4 \%)$ patients had no

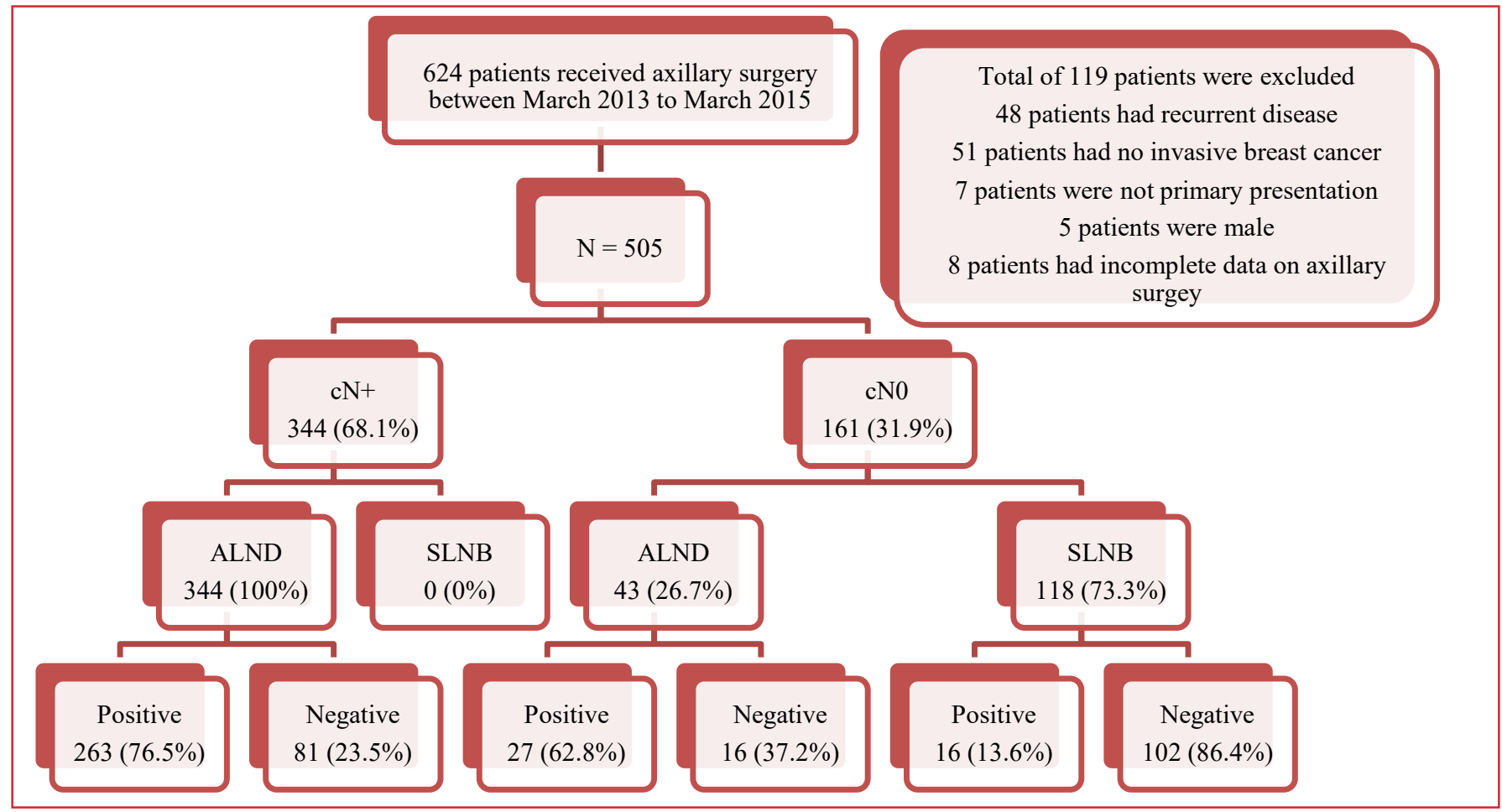

Figure 1. Flowchart of axillary surgery 
Table 2. Reasons for ALND performed in cN0 patients

\begin{tabular}{lccc}
\hline Reason for ALND & Total & Positive ALND & Negative ALND \\
& $\mathbf{N = 4 3 ( \% )}$ & $\mathbf{N}=\mathbf{2 7}(\mathbf{\%})$ & $\mathbf{N = 1 6 ( \% )}$ \\
\hline Preoperative decision - "large" tumour & $7(16.3)$ & $4(14.8)$ & $3(18.8)$ \\
Intraoperative decision - suspicious lymph nodes & $22(51.2)$ & $15(55.6)$ & $7(43.8)$ \\
No tracer or gamma probe available & $3(6.9)$ & $0(0)$ & $3(18.7)$ \\
No SLN identified intraoperatively & $1(2.3)$ & $0(0)$ & $1(6.2)$ \\
No data & $10(23.3)$ & $8(29.6)$ & $2(12.5)$
\end{tabular}

information on histological subtype. Immunohistochemistry identified $87(17.2 \%)$ patients with features of a Luminal A intrinsic subtype breast cancer, $303(60.0 \%)$ with a Luminal B subtype, $43(8.5 \%)$ were HER-2 enriched and $71(14.1 \%)$ were classified as triple-negative. One patient had no immunohistochemistry record $(0.2 \%)$. The majority of patients $(313,61.9 \%)$ underwent primary surgery and 192 (38.1\%) patients had surgery following neoadjuvant chemotherapy (Table 1).

On the basis of clinical examination and ultrasound, 344 patients were staged $\mathrm{cN}+(68.1 \%), 161$ (31.9\%) were assessed as clinically node-negative (cN0) and were eligible for SLNB. A SLNB was performed in 118 out of these $161 \mathrm{cN} 0$ patients (73.3\%). A negative SLNB was found in $102(86.4 \%)$ and a positive SLNB in $16(13.6 \%)$ patients (Figure 1).

In the cN0 group, 43 patients $(26.7 \%)$ underwent ALND, $27(62.8 \%)$ had a positive ALND and $16(37.2 \%)$ had a negative ALND. These patients underwent ALND for various reasons which include non-availability of tracer or gamma probe, "large" tumour size $(>4 \mathrm{~cm})$ and the surgeon's clinical decision intraoperatively to proceed to an ALND (Table 2).

In the $\mathrm{cN}+$ group, 263 out of the 344 patients $(76.4 \%)$ had a positive ALND and 81 (23.5\%) a negative ALND.

Overall, 306 patients were $\mathrm{pN}+(60.6 \%)$ and $199 \mathrm{pN} 0$ $(39.4 \%)$.
A total of $313(62.0 \%)$ of 505 patients underwent primary surgery and $192(38.0 \%)$ received NACT. After exclusion of patients who had NACT, 169 patients were staged as $\mathrm{cN}+$ $(54.0 \%)$ and $144(46.0 \%)$ as $\mathrm{cN} 0$ (Figure 2). In the $\mathrm{cN}+$ group undergoing primary surgery, $169(100 \%)$ underwent ALND and $36(21.3 \%)$ were found $\mathrm{pN} 0$.

The sensitivity, specificity, positive predictive value (PPV) and negative predictive value (NPV) of preoperative clinical assessment in predicting pathological status were $85.9 \%$, $59.3 \%, 76.5 \%$ and $73.9 \%$ respectively. In patients who had primary surgery, the sensitivity, specificity, PPV and NPV were $80.6 \%, 73.9 \%, 78.7 \%$ and $76.1 \%$ respectively (Table 3 ).

On univariate analysis, patients after NACT of whom only nine had a SLNB were not statistically more likely to have a pN0 ALND compared to those patients who underwent primary surgery (OR 1.06, 95\% CI: 0.7-1.7, $\mathrm{p}=0.790$ ).

\begin{tabular}{lcc} 
Table 3. Validity of clinical staging and axillary surgery \\
\hline $\begin{array}{c}\text { All patients } \\
\text { N = 505 }\end{array}$ & $\begin{array}{c}\text { Primary surgery } \\
\text { alone }(\mathbf{n}=\mathbf{3 0 3})\end{array}$ \\
\hline Sensitivity & $85.9 \%$ & $80.6 \%$ \\
Specificity & $59.3 \%$ & $73.9 \%$ \\
PPV & $76.5 \%$ & $78.7 \%$ \\
NPV & $73.9 \%$ & $76.1 \%$
\end{tabular}

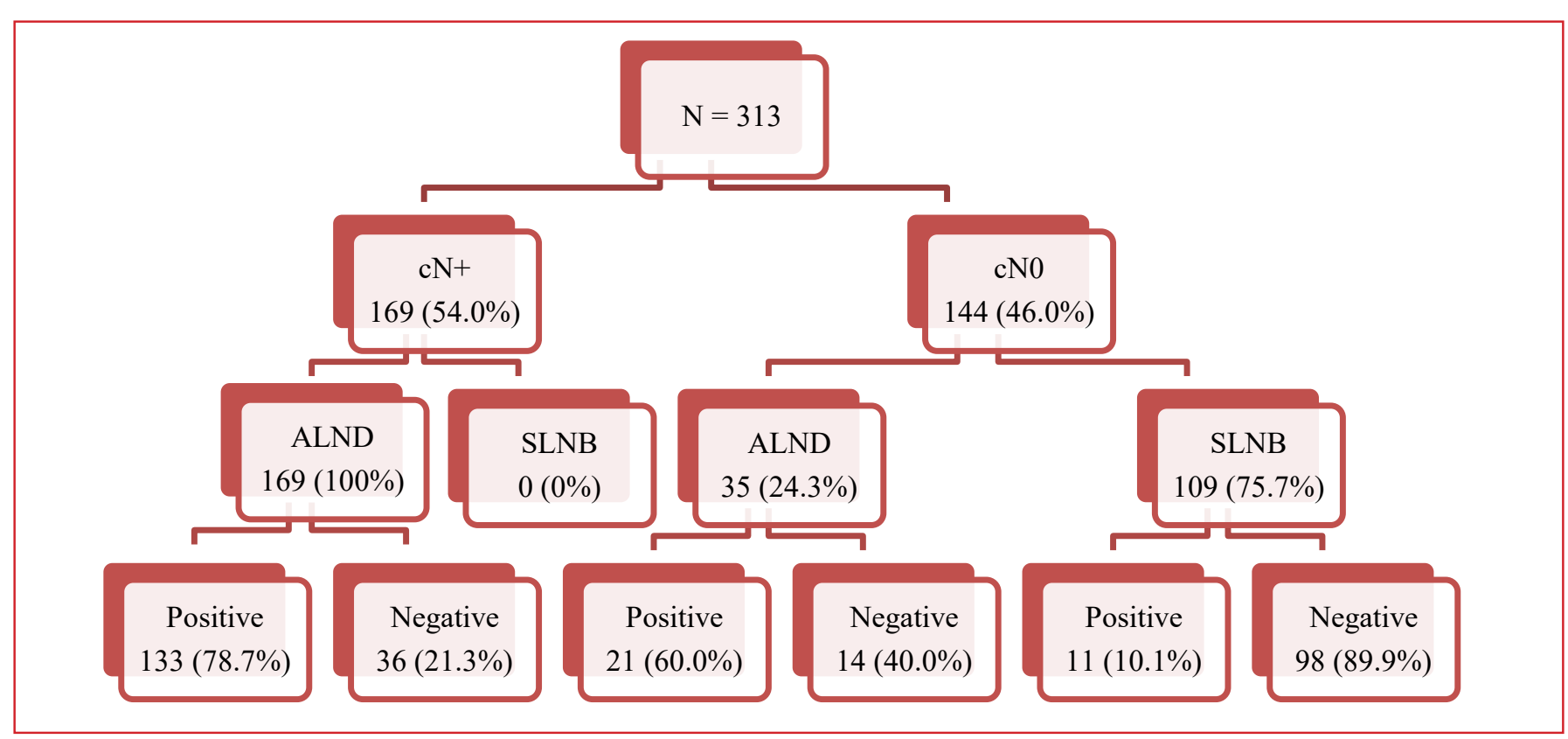

Figure 2. Flowchart of women with invasive breast carcinoma who have primary surgery 
Table 4. Univariate and multivariate analysis of factors associated with a pNO ALND

\begin{tabular}{|c|c|c|c|c|c|}
\hline Characteristics & & $\begin{array}{c}\text { Univariate analysis } \\
\text { OR }(95 \% \mathrm{CI}) \\
\end{array}$ & P-value & $\begin{array}{c}\text { Multivariate analysis } \\
\text { OR }(95 \% \mathrm{CI})\end{array}$ & P-value \\
\hline Age & & $1.00(0.99-1.02)$ & 0.467 & & \\
\hline \multirow[t]{5}{*}{ Age group } & $<40$ & Reference & 0.950 & & \\
\hline & $40-49$ & $1.11(0.5-2.4)$ & & & \\
\hline & $50-59$ & $1.24(0.6-2.7)$ & & & \\
\hline & $60-69$ & $1.36(0.6-3.0)$ & & & \\
\hline & $\geq 70$ & $1.21(0.5-3.0)$ & & & \\
\hline \multirow[t]{5}{*}{ T-stage } & $\mathrm{Tx}$ & $8.45(0.7-97.5)$ & 0.267 & & \\
\hline & $\mathrm{T} 1$ & $1.63(0.5-5.0)$ & & & \\
\hline & $\mathrm{T} 2$ & $1.56(0.9-2.7)$ & & & \\
\hline & $\mathrm{T} 3$ & $1.61(0.8-3.2)$ & & & \\
\hline & $\mathrm{T} 4$ & Reference & & & \\
\hline \multirow[t]{2}{*}{ Clinical nodal status } & No & $1.92(1.0-3.7)$ & 0.054 & $2.10(1.0-4.3)$ & 0.039 \\
\hline & N1 & Reference & & & \\
\hline \multirow[t]{2}{*}{ Clinical staging } & I \& II & $1.40(0.9-2.2)$ & 0.151 & & \\
\hline & III \& IV & Reference & & & \\
\hline \multirow[t]{2}{*}{ Histological type } & Ductal & Reference & 0.908 & & \\
\hline & Others & $1.06(0.4-3.0)$ & & & \\
\hline \multirow[t]{4}{*}{ Molecular subtype } & Luminal A & $1.02(0.5-2.0)$ & 0.003 & $1.07(0.5-2.1)$ & 0.849 \\
\hline & Luminal B & Reference & & Reference & Reference \\
\hline & HER-2 enriched & $2.05(1.0-4.3)$ & & $2.25(1.1-4.8)$ & 0.035 \\
\hline & Triple-negative & $2.98(1.6-5.5)$ & & $3.05(1.6-5.7)$ & 0.001 \\
\hline \multirow[t]{2}{*}{ First treatment } & Surgery & Reference & & & \\
\hline & $\begin{array}{l}\text { Neoadjuvant } \\
\text { chemotherapy }\end{array}$ & $1.06(0.7-1.7)$ & 0.790 & $1.10(0.6-1.7)$ & 0.964 \\
\hline \multirow{2}{*}{$\begin{array}{l}\text { Preoperative biopsy of } \\
\text { the axilla }\end{array}$} & No & $1.71(0.7-4.0)$ & 0.195 & & \\
\hline & Yes & Reference & & & \\
\hline
\end{tabular}

Patients without preoperative biopsy of the axilla were also not found more likely to have a pN0 ALND compared to patients who had preoperative biopsy of the axilla, but numbers were small. Only 40 patients had a preoperative biopsy of the axilla (10.39\%) (OR 1.71, 95\% CI: 0.7-4.0, p = 0.195). Triplenegative and HER-2 enriched patients were more likely to have a negative ALND than those with luminal subtypes (OR 3.05, 95\% CI: 1.6-5.7, $\mathrm{p}=0.001$ and OR $2.25,95 \% \mathrm{CI}$ : 1.1-4.8, $\mathrm{p}=0.035)$. $\mathrm{cN} 0$ was associated with an increased risk compared $\mathrm{cN}+(\mathrm{OR} 2.10, \mathrm{CI} 95 \%$ : 1.0-4.3, $\mathrm{p}=0.039)$. Age, tumour size, histological type and preoperative biopsy of the axilla were not associated with negative ALND (Table 4).

\section{Discussion}

A quarter of our patients $(97,25.06 \%)$ who underwent an ALND were ultimately node-negative on pathology. Node dissection has no oncological benefit in the nodenegative patient and is associated with complications such as lymphoedema. Overtreatment of the axilla is regarded as obsolete in modern breast cancer surgery and European guidelines have set standards on the proportion of pN0 patients who did not undergo ALND with the minimum standard set at $80 \%$ and the target at $90 \% .^{12}$ These standards were not reached in our cohort; only $66.2 \%$ were spared ALND after exclusion of patients who received NACT.

Various factors need to be considered.

Firstly, 81 of the 97 pN0 patients were initially assessed as clinically node-positive $(83.5 \%)$. Very few of our patients had preoperative histopathological confirmation of nodal involvement and the clinical assessment in this cohort had a specificity of $59.3 \%$ and PPV of $76.5 \%$. NACT may alter the nodal status but exclusion of patients who received NACT improved these values only to $73.9 \%$ and $78.7 \%$. This highlights the limitations of our preoperative assessments. We were not able to demonstrate statistical significance for risk without preoperative attempted pathology confirmation but numbers were small and lacked statistical power. Negative predictive value of clinical assessment was equally limited at $73.9 \%$ and $76.1 \%$ after exclusion of patients who received NACT. Preoperative clinical assessment needs to be improved to allow for appropriate choice of surgical axillary approach. A 
future audit of axillary ultrasounds may assist to improve the accuracy of preoperative staging of the axilla in our practice.

Secondly, ALND was performed in 43 clinically nodenegative patients for various reasons such as large tumour size, intraoperative impression of nodal involvement or non-availability of a gamma-probe or radio-colloid tracer. In 15 out of 22 cases the intraoperative decision to perform an ALND was correct and in 4 out of 7 cases where the decision was taken to proceed to an ALND, the axilla was found to have involved lymph nodes. Although 16 (37.2\%) were pathologically node-negative, these clinical decisions to proceed with ALND need to be viewed in the context of a resource-constrained environment. On the one hand, the majority $(62.8 \%)$ were ultimately $\mathrm{pN}+$ and a SLNB would have potentially required a second surgery to perform a completion ALND. On the other hand, SLNB should be offered in a well-resourced setting to patients with large breast cancers and the intraoperative impression of node involvement confirmed by intraoperative pathology before proceeding to ALND. Intraoperative pathology is currently not routinely available. A hypothetical application of SLNB in these cases would have increased the proportion of pathologically nodenegative patients who did not undergo ALND to $77 \%$, close to the European minimum standard of $80 \% .^{12}$ Under our circumstances we need to find an appropriate balance to have both a cost-effective and safe policy to manage the axilla in breast cancer.

The National Surgical Adjuvant Breast and Bowel Project (NSABP) Trials B-18 and B-27 demonstrated that NACT can downstage $30-40 \%$ of clinically node-positive patients with no residual disease found in the axilla at the time of surgery. ${ }^{13,14}$ The application of SLNB for initially nodepositive patients after neoadjuvant chemotherapy has been evaluated in the ACOSOG Z1071 and SENTINA trials. ${ }^{15,16}$ A FNR of under $10 \%$ can be achieved by using a dual-tracer technique and identifying at least three sentinel nodes. In a recent prospective study, $68 \%$ of initially pathologically confirmed node-positive patients became candidates for SLNB after NACT and an ALND could be avoided in $48 \% .{ }^{17}$ This opens up the possibility of avoiding ALND after NACT in a subset of our patients. During the study period, we did not yet apply this approach and the nine patients who underwent SLNB after NACT were initially cN0.

Complete pathologic response rates vary among intrinsic subtypes and are reportedly higher in hormone receptor negative tumours. We demonstrated a significantly higher risk for a pathologically node-negative ALND in the triplenegative and HER-2 enriched subtypes.

47 of 183 patients in this study who received NACT and ALND had a pN0 axillary dissection (25.68\%). It must be recognised that most patients did not have histological or cytological confirmation of axillary involvement before NACT, and therefore the pre-treatment assessment of the axilla was not in keeping with international standards. In addition, a pathologic complete response was found in only 11 cases out of the $192(5.7 \%)$ patients who were operated following NACT, a rate considerably lower than what would be expected from the literature.

Clinical staging of this cohort is skewed towards lower stage compared to the stage at presentation of our general patient population. This is explained by the inclusion of patients who received axillary surgery in this study. Our units do not operate on stage 4 disease unless limited to bone metastases or visceral oligometastases responsive to NACT. This protocol applies to this cohort although we do not have detailed data on each patient in this group.

HIV status was not recorded in this study but it is likely to affect clinical assessment of lymph nodes. Benign lymphadenopathy is often clinically and sonographically misinterpreted as neoplastic spread. BMI (body mass index) has a potential influence on axillary assessment and surgery but was not recorded in this study. Other limitations of this study include the retrospective design, the often incomplete nature of clinical records and the relatively small number of patients.

\section{Conclusion}

More than a quarter $(25.06 \%)$ of axillary node dissections were performed for pathologically node-negative disease in our study population.

Whilst SLNB is still being underutilised, only 16 out of 118 actually performed SLNBs were node-positive. Overall, 199 patients had a pathologically negative axilla and this shows that there is ample space for improvement.

Two interventions can address this situation and help to achieve international standards. Firstly, the outdated preoperative nodal assessment needs to be improved. Routine confirmation of nodal involvement by cytology or histology will increase accuracy of clinical staging and improve selection for SLNB.

Broader application of SLNB would increase the rate of pathological node-negative patients who did not receive ALND to $77 \%$, close to targets set in well-resourced countries. We need to find an appropriate balance and have both a costeffective and safe policy to manage the axilla in breast cancer.

Secondly, we need to expand SLNB to patients after NACT. In keeping with new international guidelines, these patients should also have preoperative imaging and biopsy before being treated as clinically node-positive. Bearing in mind the specific requirements of using two tracers and harvesting three nodes they should no longer be denied the option of SLNB.

HIV-associated lymphadenopathy requires particular attention since it may confound clinical nodal assessment and its effect is currently being evaluated in a prospective study.

\section{Declarations of conflict of interest}

The authors declare no conflict of interest.

\section{Funding source}

None 


\section{Orcid}

C Groenewald (iD http://orcid.org/0000-0002-7279-3797

H Cubasch (iD http://orcid.org/0000-0001-6110-3984

A Mannell (iD) http://orcid.org/0000-0003-4438-4045

O Ayeni (D) http://orcid.org/0000-0002-1132-2860

S Nietz (D) http://orcid.org/0000-0002-6313-2794

\section{REFERENCES}

1. Feig BW, Ching CD, Christine D. University of Texas M.D. Anderson Cancer Center. Department of Surgical Oncology. The M.D. Anderson surgical oncology handbook [Internet]. Wolters Kluwer/Lippincott Williams \& Wilkins; 2012 [cited 2018 May 6]. 900 p. Available from: http://www.ovid.com/site/ catalog/books/3552.jsp

2. Lee MC, Joh JE, Chau A. Axillary Staging Prior to Neoadjuvant Chemotherapy: The Roles of Sentinel Lymph Node Biopsy and Axillary Ultrasonography. Cancer Control [Internet]. 2012 Oct [cited 2018 May 6];19(4):277-85. Available from: http://www. ncbi.nlm.nih.gov/pubmed/23037495

3. Valente SA, Levine GM, Silverstein MJ, Rayhanabad JA, Weng-Grumley JG, Ji L, et al. Accuracy of Predicting Axillary Lymph Node Positivity by Physical Examination, Mammography, Ultrasonography, and Magnetic Resonance Imaging. Ann Surg Oncol [Internet]. 2012 Jun 7 [cited 2018 May 6];19(6):1825-30. Available from: http://www.ncbi.nlm. nih.gov/pubmed/22227922

4. Cubasch H, Joffe M, Hanisch R, Schuz J, Neugut AI, Karstaedt A, et al. Breast cancer characteristics and HIV among 1,092 women in Soweto, South Africa. Breast Cancer Res Treat. 2013;140(1):177-86.

5. Houssami N, Ciatto S, Turner RM, Cody HS, Macaskill P. Preoperative Ultrasound-Guided Needle Biopsy of Axillary Nodes in Invasive Breast Cancer. Ann Surg [Internet]. 2011 Aug [cited 2018 May 6];254(2):243-51. Available from: http://www. ncbi.nlm.nih.gov/pubmed/21597359

6. Mittendorf EA, Hunt KK, Boughey JC, Bassett R, Degnim AC, Harrell R, et al. Incorporation of Sentinel Lymph Node Metastasis Size Into a Nomogram Predicting Nonsentinel Lymph Node Involvement in Breast Cancer Patients With a Positive Sentinel Lymph Node. Ann Surg [Internet]. 2012 Jan [cited 2018 May 6];255(1):109-15. Available from: http://www. ncbi.nlm.nih.gov/pubmed/22167004

7. Norman SA, Localio AR, Kallan MJ, Weber AL, Torpey HAS, Potashnik SL, et al. Risk factors for lymphedema after breast cancer treatment. Cancer Epidemiol Biomarkers Prev [Internet]. 2010 Nov [cited 2018 May 6];19(11):2734-46. Available from: http://www.ncbi.nlm.nih.gov/pubmed/20978176

8. Krag DN, Anderson SJ, Julian TB, Brown AM, Harlow SP, Ashikaga T, et al. Technical outcomes of sentinel-lymph-node resection and conventional axillary-lymph-node dissection in patients with clinically node-negative breast cancer: results from the NSABP B-32 randomised phase III trial. Lancet Oncol
[Internet]. 2007 Oct [cited 2018 May 6];8(10):881-8. Available from: http://www.ncbi.nlm.nih.gov/pubmed/17851130

9. NCCN. National Comprehensive Cancer Network. Breast Cancer. Version 1.2018. In 2018.

10. Goldhirsch A, Wood WC, Coates AS, Gelber RD, Thürlimann B, Senn H-J, et al. Strategies for subtypes-dealing with the diversity of breast cancer: highlights of the St Gallen International Expert Consensus on the Primary Therapy of Early Breast Cancer 2011. Ann Oncol [Internet]. 2011 Aug 1 [cited 2018 May 6];22(8):1736-47. Available from: http://www. ncbi.nlm.nih.gov/pubmed/21709140

11. Dialani V, James DF, Slanetz PJ. A practical approach to imaging the axilla. Insights Imaging [Internet]. 2015 Apr [cited 2019 Apr 26];6(2):217-29. Available from: http://www.ncbi. nlm.nih.gov/pubmed/25534139

12. Del Turco MR, Ponti A, Bick U, Biganzoli L, Cserni G, Cutuli B, et al. Quality indicators in breast cancer care. Eur J Cancer [Internet]. 2010 Sep [cited 2018 May 6];46(13):2344 56. Available from: http://www.ncbi.nlm.nih.gov/ pubmed 120675120

13. Fisher B, Brown A, Mamounas E, Wieand S, Robidoux A, Margolese RG, et al. Effect of preoperative chemotherapy on local-regional disease in women with operable breast cancer: findings from National Surgical Adjuvant Breast and Bowel Project B-18. J Clin Oncol [Internet]. 1997 Jul [cited 2018 May 6];15(7):2483-93. Available from: http://www.ncbi.nlm.nih. gov/pubmed/9215816

14. Bear HD, Anderson S, Brown A, Smith R, Mamounas EP, Fisher B, et al. The Effect on Tumor Response of Adding Sequential Preoperative Docetaxel to Preoperative Doxorubicin and Cyclophosphamide: Preliminary Results From National Surgical Adjuvant Breast and Bowel Project Protocol B-27. J Clin Oncol [Internet]. 2003 Nov 15 [cited 2018 May 6];21(22):4165-74. Available from: http://www.ncbi.nlm.nih. gov/pubmed/14559892

15. Boughey JC, Suman VJ, Mittendorf EA, Ahrendt GM, Wilke LG, Taback B, et al. Sentinel Lymph Node Surgery After Neoadjuvant Chemotherapy in Patients With Node-Positive Breast Cancer. JAMA [Internet]. 2013 Oct 9 [cited 2018 May 6];310(14):1455. Available from: http://www.ncbi.nlm.nih.gov/ pubmed/24101169

16. Kuehn T, Bauerfeind I, Fehm T, Fleige B, Hausschild M, Helms $\mathrm{G}$, et al. Sentinel-lymph-node biopsy in patients with breast cancer before and after neoadjuvant chemotherapy (SENTINA): a prospective, multicentre cohort study. Lancet Oncol [Internet]. 2013 Jun [cited 2018 May 6];14(7):609-18. Available from: http://www.ncbi.nlm.nih.gov/pubmed/23683750

17. Mamtani A, Barrio AV, King TA, Van Zee KJ, Plitas G, Pilewskie M, et al. How Often Does Neoadjuvant Chemotherapy Avoid Axillary Dissection in Patients With Histologically Confirmed Nodal Metastases? Results of a Prospective Study. Ann Surg Oncol [Internet]. 2016 Oct 9 [cited 2018 May 6];23(11):3467-74. Available from: http://www.ncbi.nlm.nih. gov/pubmed/27160528 